\title{
USE OF ENVIRONMENTAL PSYCHOLOGY AND VIRTUAL REALITY FOR A USER-CENTERED DESIGN APPROACH
}

\author{
GIUSEPPE MARTINO DI GIUDA ${ }^{1}$, SIMONE PRADELLA ${ }^{2}$, and MARCO SCHIEVANO ${ }^{2}$ \\ ${ }^{I}$ Dept of Management, Università Degli Studi di Torino, Torino, Italy \\ ${ }^{2}$ Dept of Architecture, Built Environment and Construction Engineering, Politecnico di \\ Milano, Milano, Italy
}

\begin{abstract}
This paper presents a method to integrate qualitative requirements in the design process, starting from environmental psychology, and including the use of virtual reality systems. The current approach to the design process is mainly based on compliance with building codes or regulations and developed through consolidated practices or previous expertise. The goal of this research is the creation of a work method, based on scientific evidences, that puts the user at the center of the process. Focused on school buildings and through a critical review, four main themes are defined as guidelines for the designer: a) perception of the building; b) physical characteristics; c) design of the classroom space and furniture; d) school green spaces and outdoor activities. These represent the starting point, potentially improved through the definition of new fields of research; for this purpose, it is possible evaluate the use of VR technologies as a potential support tool. The paper also proposes an experimental model to investigate, by VR technologies, the effects related to exposure to the external environment from the internal one, by studying two variables: the type of connection (visual vs. physical) and the type of environment (natural vs. anthropic) in relation to student's stress and attention levels. This approach provides a new vision of the design phase where the focus is brought to the user through the analysis of scientific evidence, together with the use of new technologies.
\end{abstract}

Keywords: Experimental model, Immersive reality, Scientific evidence, Users' needs, Design assessment.

\section{INTRODUCTION}

\subsection{Premise}

Our living environment plays a fundamental role in everyday life. A reality that is able to influence body and mind. For this reason, space users must be increasingly placed at the center of the project's design (Ulrich 1984). The search of a user-centered environment does not prejudice aesthetic and/or functional aspects, but instead represents a challenge for the designers, who are called to balance these factors. To do this, the identification of a replicable method is necessary in order to keep into account the several factors involved. The adoption of a design approach based on scientific evidence (EBD) represents a possible solution (Sailer et al. 2008). This practice implicates a critical analysis of the bibliography regarding the project's theme to be made by the designer, who extracts information and/or requirements set as fixed points during the design phase. This, unlike the design based on consolidated practices, favors a constant search 
for efficiency and design effectiveness in relation to the needs of individual users, suitably integrated with the client's wishes.

\subsection{Requirements}

The process requires two main elements: an application context (Sailer et al. 2008) and a strict scientific evidence analysis protocol (Pawson 2002). The first one allows the definition of the boundary conditions and could represent, according to the literature, a limiting factor in presence of realities characterized by different management solutions (e.g., offices). The second one ensures high level of validity and provides the main important steps necessary to the extraction and the systematization of the scientific evidence collected in relation to the formulated areas of investigation. These steps can be integrated by the introduction of an active validation of the proposed choices supported by the use of immersive Virtual Reality technologies (VR) ${ }^{1}$ insert in an experimental study.

\subsection{Goals}

Considering the premises, it is possible define a primary goal as the creation of a work method based on scientific evidence, that puts the user at the center of the process, analyzing the effects produced by the environment. A work method that can improve, as a secondary goal, the evaluation of VR as a potential support tool for this purpose. Both refer to a hypothetical case, reported as a guideline.

\section{METHOD}

\subsection{General Approach}

The general approach provides the definition of the work method proposed. Presented as a guideline, it includes 3 main steps:

- Definition of an evidence repertory;

- Identification of potential developments and research activity;

- Design application.

These steps, analyzed below, are replicable for each context considered. In this paper, the context is that of a primary school, as it allows the analysis of different management solutions like different didactic models, and at the same time, it allows to evaluate the effects of the built environment on a sensitive category of users.

\subsection{Definition of an Evidence Repertory}

The first step provides a definition of an evidence repertory conducted according to the scientific evidence analysis protocol. It affects the whole process with the definition of the main field of investigation and the analysis of the evidence. Related to the school context, it is possible to propose the following areas of investigation:

- Effects due to the perception of the building;

- Effects due to the physical characteristics of the school environment;

\footnotetext{
${ }^{1}$ Virtual reality completely displaces a person to another location. It obscures the user's physical surroundings and replaces them with a computer-generated scene or one that was previously captured (Brigham 2017).
} 
- Effects due to the design of the indoor space and the choice of furniture;

- Effects of school green spaces and outdoor activities.

These, inspired by the structure adopted by the bibliographic review carried out by Manca et al. (2020), facilitate the interpretation of environmental factors and cognitive effects.

\subsection{Setting of Development and Research Activity}

Based on research activity and an extension of bibliography reference, it is possible to provide a definition of a method to further case studies. According to the secondary goal of this paper, it is possible define an integration of the approach with the use of VR technologies. This approach, based on the analysis of the effects caused by the connection between the class space and the external environment, is developed in 3 main elements: hypotheses of the study, participants and statistical parameters, procedure. The first one represents a core moment of the design of the experimental study. In relation to the use of VR it is necessary to identify a field of study that can be investigated through an environmental simulation. For this reason, it is possible to adopt, as example, the following hypotheses:

- The presence of external natural environments can influence the levels of attention;

- The surrounding context has different cognitive effects depending on the kind of connection (visual vs. physical).

The second main element defines the statistical requirement. For the hypothetical study it is possible refer to the following parameters:

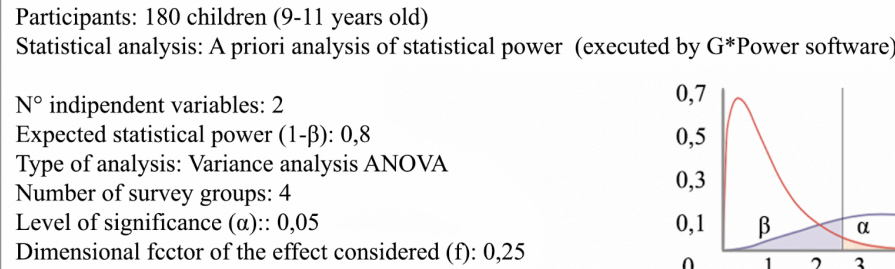

Figure 1. Statistical parameters.

The last one element requires the definition of the procedures of the VR experience and the necessary tool according to the objectives sought. The experience planned in a controlled environment with natural or artificial simulated contest, according to the experimental design proposed, provide the following procedures.

Table 1. Steps, materials, procedure take from the proposed hypothetical study.

\begin{tabular}{|c|c|c|}
\hline Phases & Procedures & Tools \\
\hline $1 d=10 \min$ & $\begin{array}{l}\text { Virtual environment } \\
\text { exploration }\end{array}$ & $\begin{array}{l}\text { CAVE, a fully immersive virtual reality environment that } \\
\text { simulate controlled contexts (Manjrekar et al. 2014). }\end{array}$ \\
\hline $2 d=1 h$ & Test & Non-verbal logic, creativity and comprehension tests. \\
\hline $3 d=5 \min$ & Cortisol level monitoring & Analysis of the salivary level of cortisol. \\
\hline $4 d=10 \mathrm{~min}$ & Attentive skills analysis & Orientation and attention test. \\
\hline
\end{tabular}


These procedures allow the analysis of the factors considered during the evaluation of the influence produce by the environment and the kind of its connection.

\subsection{Design Application}

Refocus the attention to the main steps of the general approach, it is necessary present the last one: the design application. This step provides the translation of the evidence extracted from the literature and the experimental study. This operation requires a design sensibility and the work of the designer called to balance these elements with aesthetic/functional factors.

\section{RESULTS}

The proposed method allows obtaining distinct results in relation to the progressive development of the process. For this reason, it is possible present the results on the base of the pervious steps.

\subsection{Evidence Repertory}

The first results obtained by the definition of a reference repertory, it is a collection of evidence potentially useful during the design phase as a starting point to put the user needed at the center of the project. In relation to the school context, the evidences are structured according to the fields of investigation propose. In order to provide a synthetic treatment, the following summary table is provided.

Table 2. Reference repertory.

Perception of the building

Qualitative elements perceived by users: -quality of construction provide a positive perception (Stringer et al. 2012)

\section{Aspects of the space: -an easy reading space (flexible, with reserved spaces and interaction area) facilitates cognitive development (Slunjski 2015)}

Age of the building: -recently constructed buildings generate an increase in performance of 2.5-7.7\% (Bowerws and Burkett 1989)

\section{Physical characteristics of school environment}

Acoustic characteristics: -a noise level $<35 d B$ (unoccup. class), a reverb. time $<0,4 s$, and $a$ pupil-teacher distance $<12 m$ improve learning (Whitlock and Dodd 2008)

\section{Environmental} characteristics: - $t 20^{\circ} \mathrm{C}$, and a humid. 50$55 \%$ improve learning (Wargocki and Wyon 2007)

Lighting characteristics: - an illuminance level of the studio floor 300 lux improves cognitive process (Sleegers et al. 2013)
Design of classroom space and furniture

Spatial characteristics and arrangement of furniture:

-group seating not to define optimal learning and shaded areas (Park and Choi 2014) -simple layouts favour the learning of children, especially those suffering from difficulties

(McAllister 2012)

Characteristics of the furniture:

-modular furniture allow

the regulation of the cooperation (Rosenfield et al. 1985)
School green spaces and outdoor activities

Characteristics of green spaces:

-maintained environments foster the desire for cooperative activities (Fjørtoft 2001) -the presence of different elements of interaction (trees, stones, ponds) reduce stress and improve cognitive processes (Fjørtoft 2001)

Presence of green spaces:

-view and interaction with green space reduce distractions during class hours (Chawla et al. 2014) 


\subsection{Evidence Obtained by the Experimental Research Activity}

According to the method it is possible expect, as a result, an improvement of the evidence repertory by the result of the experimental research activity. In relation to the previous hypothesis provide, not tested, it is possible to identify the following expected results.

\begin{tabular}{|c|c|c|c|}
\hline \multirow{3}{*}{ Expected result } & \multicolumn{2}{|r|}{ Applicative relevance } & \multirow[b]{2}{*}{ Antropic contest } \\
\hline & Contest & $\underline{\text { Natural contest }}$ & \\
\hline & Natural Antropic & 1) Increased glass surfaces & Re-naturalisation of \\
\hline Direct access & ++ & and interior-exterior & courtyards and school \\
\hline No direct acc. & + & connection. & $\begin{array}{l}\text { context. Limitation of the } \\
\text { number of direct connect. }\end{array}$ \\
\hline
\end{tabular}

Figure 2. Expected results and applicative relevance.

These results, expected according to the existing bibliography (Kaplan and Talbot 1983), represent an example of the further areas of investigation useful for underline the possible extension of the work method and how VR experience can be considered as a valid tool.

\subsection{Design Application}

The last possible results, related to the design solution, required a reference to a hypothetical building. For this reason, it is possible to refer the following analysis to a hypothetical classroom with a non-linear geometry in the plane and a roof exposed to only one wing, contiguous with other classrooms. This starting point can be improved through the application of the evidences emerged, by adopting the following actions:

Table 3. Intervention and expected results.

\section{Intervention}

Adoption of a movable partition to separate two classrooms.

Introduction of air condition. systems.

Introduction of a curtain wall system to replace the irregular perimeter portion

Introduction of an internal suspended ceiling to balance the volume of the class.

\section{Expected results}

An interclass space perceived as safe by children -> Increased levels of socialization.

Thermal comfort -> Increased logical capacity.

An understandable space and an improvement of the connection indoor/outdoor -> Reduction of stress levels,

An understandable space -> Reduction of stress levels, increase in attentive capacity.

These interventions can increase the levels of wellbeing/comfort/learning and highlight the direct correlation between evidence and intervention.

\section{DISCUSSION AND CONCLUSION}

\subsection{Correlation Between Results and Objectives}

Analyzing the correlation between result and objectives, it is possible to highlight the potential impact in terms of increased levels of learning and well-being, deriving from an evidence-based approach. These reflections put the user at the center of the process and highlight the possible 
wide use of this approach. The use of VR technologies also represents a promising tool to support the research of new evidence according to a replicable method.

\subsection{Limitations}

Observing the approach proposed, it is possible define as a limiting factor the actual absence of a collaborative validation of the evidence. The introduction of a reference framework/database constantly updated can help in solving this issue. This approach can facilitate the improvement of the design process and represent the union point between aesthetic, functional and well-being aspects of individual users.

\section{References}

Bowerws, J. H., and Burkett, C. W., Effect of Physical and School Environment on Students and Faculty, Educational Facility Planner, Council of Educational Facility Planner, 27(1), 28-29, February, 1989.

Brigham, T. J., Reality Check: Basics of Augmented, Virtual, and Mixed Reality, Medical Reference Services Quarterly, Taylor and Francis Ltd, 36(2), 171-178, April, 2017.

Chawla, L., Keena, K., Pevec, I., and Stanley, E., Green Schoolyards as Havens from Stress and Resources for Resilience in Childhood and Adolescence, Health \& Place, Elsevier, 28, 1-13, July, 2014.

Fjørtoft, I., The Natural Environment as a Playground for Children: The Impact of Outdoor Play Activities in Pre-primary School Children, Early Childhood Educational Journal, 29(2), 111-117, January, 2001.

Kaplan, S., and Talbot, J. F., Psychological Benefits of a Wilderness Experience, Behavior and the Natural Environment, Springer, 6, 163-203, 1983.

Manca, S., Cerina, V., Tobia, V., Sacchi, S., and Fornara, F., The Effect of School Design on User's Responses: A Systematic Review, Sustainability, 12(8), 3453, April, 2020.

Manjrekar, S., Sandilya, S., Bhosale, D., Kanchi, S., Pitkar, A., and Gondhalekar, M., CAVE: An Emerging Immersive Technology - A Review, $16^{\text {th }}$ International Conference on Computer Modeling and Simulation, Cambridge, UK, March, 2014.

McAllister, K., Design Considerations for the Autism Spectrum Disorder-Friendly Key Stage 1 Classroom, Support for Learning, Nasen, 27(3), 103-113, August, 2012.

Park, E. L., and Choi, B. K., Transformation of Classroom Spaces: Traditional vs Active Learning Classroom in Colleges, Higher Educational, Springer, 68(5), 749-771, November, 2014.

Pawson, R., Evidence-Based Policy: in Search of a Method, Evaluation, SAGE, 8, 157-181, April, 2002.

Rosenfield, P., Lambert, N. M., and Black, E. A., Desk Arrangement Effects on Pupil Classroom Behaviour, Journal of Educational Psychology, APA, 77(1), 101-108, February, 1985.

Sailer, K., Budgen, A., Lonsdale, N., Turner, A., and Penn, A., Evidence-Based Design: Theoretical and Practical Reflections of an Emerging Approach, $4^{\text {th }}$ Design Research Society Conference, Sheffield, UK, July, 16-19, 2008.

Sleegers, P. J. C., Moolenaar, N. M., Galetzka, M., Pruyn, A., Sarroukh, B. E., and van der Zande, B., Lighting Affects Students' Concentration Positively: Findings from Three Dutch Studies, Lighting Research \& Technology, SAGE, 45(2), 159-175, June, 2013.

Slunjski, E., Multidisciplinary Approach to Designing Space of Early Childhood Education Institution as a Condition for High-Quality Education Process, Croatian Journal of Education, 17(1), 253-264, March, 2015.

Stringer, A., Dunne, J., and Boussabaine, H., School design quality: A user perspective, Architectural Engineering \& Design Management, Taylor and Francis Ltd., 8(4), 257-272, August, 2012.

Ulrich, R., View Through a Window may Influence Recovery from Surgery, Science, AAAS, 224, 420-421, April, 1984.

Wargocki, P., and Wyon, D. P., The Effects of Moderately Raised Classroom Temperatures and Classroom Ventilation Rate on the Performance of Schoolwork by Children, HVAC\&R Research, ASHRAE, 13(2), 193-220, March, 2007.

Whitlock, J. A. T, and Dodd, G., Speech Intelligibility in Classrooms Specific Acoustical Needs for Primary School Children, Building acoustics, SAGE, 15(1), 35-47, January, 2008. 